\title{
Water drops bouncing off vertically vibrating textured surfaces
}

\author{
Wei Wang ${ }^{1}$ Chen $\mathrm{Ji}^{1,3}$ Fangye Lin ${ }^{1}$ Jun Zou ${ }^{1} \dagger$, and S. Dorbolo \\ ${ }^{1}$ State Key Laboratory of Fluid Power and Mechatronic Systems, Zhejiang University, \\ Hangzhou 310027, China \\ ${ }^{2}$ GRASP, Physics Department B5, University of Liège - B-4000 Liège, Belgium \\ ${ }^{3}$ Institute of Marine Science and Technology, Shandong University, Qingdao 266237, China
}

(Received xx; revised xx; accepted xx)

We investigate the conditions that determine the detachment of a water drop from different vibrating textured plates by using vertical vibrations. The plate surfaces were patterned by a lattice of pillars of different shapes with different geometrical arrangements. The acceleration threshold for the water droplet to bounce off the surfaces was measured as a function of the excitation frequency. In each case, the acceleration threshold presents a minimum at the natural frequency of the droplet. The minimum acceleration required for the take-off is larger for small droplets than for large droplets. Namely, one finds that the value of the threshold depends on the size of the droplet and on the maximum apparent contact area between the droplet and the substrate. The theoretical model takes into account the energy necessary to break the capillary bridges between the droplet and the pillars of the surface. This model captures the main ingredients explaining the drop size dependence of the acceleration threshold for the take-off.

\section{Key words: droplets - vertical vibration- take-off -superhydrophobic surface}

\section{Introduction}

Super-hydrophobicity is a property that is more often than not desired by surface engineers regarding the large panel of applications: self-cleaning, anti-icing, corrosion protection (Khojasteh et al. (2016)). Classically, the superhydrophobicity property of the surface is obtained by gathering two elements: the natural water repellency due to the chemical nature of the solid and the texturation of the surface by a microstructure pattern. This controlled rugosity allows to enhance the chemical hydrophobic property of the substrate (de Gennes et al. (2008)). When a droplet interacts with a superhydrophobic substrate, according to the energy involved in the impact process, two main scenarii are observed: either the droplet remains in one piece for low speeds or the droplet splashes for high speeds. In the end, one is interested in the final state. Does the liquid adhere or not to the surface? Indeed, the droplets are found only in two states after the impact: the droplet may stick to the surface (Wenzel state) or remain very mobile (Cassie-Baxter state) (Quéré \& Reyssat (2008)). In the latter, the droplet remains at the top of the structure and in the former, the droplet is impaled in the microstructure of the surface. In the Cassie-Baxter state, even if the contact is minimized which confers the high mobility to the droplet, the adhesion to the structure remains an issue in many applications (Smith

$\dagger$ Email address for correspondence: junzou@zju.edu.cn 
et al. (2013); McBride et al. (2018)). On the superhydrophobic surfaces, the bouncing of the droplet is observed for low impact speeds. The droplet does not split and can rebound on the surface. This process has been largely investigated since the seminal work by Richard et al (Richard \& Quéré (2000)). In this regime, the water drop actually touches only the top of the pillars making the rugosity. The liquid-solid contact being minimum, the dissipation significantly decreases, leading to a high restitution coefficient $(\sim 0.9)$ that is defined as the ratio between the speeds before and after the collision (Richard et al. (2002)). Let us note that without any liquid-solid contact, the restitution coefficient is found as large as $\sim 0.95$ (de Ruiter et al. $(2014,2015)$ ); the situation is very similar to the bouncing droplet on a vertically vibrating oil bath (Couder et al. (2005); Gilet et al. (2008)). The restitution coefficient decreases for less hydrophobic surfaces, since the impalement is more and more possible increasing the solid-liquid contact area and thus increasing the dissipation (Mao et al. (1997); Bartolo et al. (2005)). To test the mobility or to confer some mobility to the droplet, one strategy is to vibrate the substrate. This strategy also applies to bubble (Zawala et al. (2011)). Horizontal vibrations can be used as an energy source in order to manipulate water droplets on the solid surfaces, even uphill (Daniel et al. (2005); Brunet et al. (2007)). On the other hand, vertical vibrations can be used to deform the droplet according to its different eigenmode (Noblin et al. (2004)). On top of that, the vertical vibrations allow controlling the mixing of the liquid inside the water droplet (Kim \& Hee-Chang (2015)). On the whole, the vertical vibrations remain an interesting mechanism to detach a droplet from the substrate. In Ref. (Boreyko \& Chen (2009); Wei et al. (2014)), droplets in Wenzel state on a substrate were vertically shaken. When the vibration amplitude is sufficient, the transition from Wenzel to Cassie-Baxter state quickly leads to the detachment of droplets. The acceleration threshold for the transition is found to depend on the excitation frequency and is found to be minimum at the natural resonance mode of the droplet. However, the acceleration remains large, about 3 times the Earth gravity. This large acceleration probably screens the transition from Cassie-Baxter to bouncing. This statement is supported by a recent paper by Raufaste et al, concerning the catapulting of water drops out of a superhydrophobic plate when the drops are initially in Cassie-Baxter state (Raufaste et al. (2017)). Indeed, the adhesion of the droplet and the plate is so small (let us remind that the coefficient of restitution is about 0.95 ) that an acceleration slightly over the Earth gravity should be sufficient for the take-off (Richard \& Quéré (2000)). On top of that, Raufaste et al demonstrated that the droplet can also benefit from a resonant mechanism with the waves generated during the acceleration to take off. In this paper, we study what should be the vertical acceleration of the plate required to see a droplet detaching from a superhydrophobic substrate when the droplet is initially in the Cassie-Baster state. Indeed, starting with a droplet in the Cassie-Baxter state allows to determine the role of the adhesion between the droplet and the top of the pillars. The acceleration threshold that is responsible for the transition between Cassie-Baxter to bouncing is to be determined in various conditions: the size of the droplet is tuned and different substrates are tested. Then, we classically model the droplet as a spring to explain the observations.

\section{Experimental results}

The droplet with a controlled and determined diameter $D$ between 2.02 and $2.62 \mathrm{~mm}$ was gently released at the surface of the substrate. The shaker was driven by a sinusoidal signal with a controlled frequency $f$. The amplitude $A$ of the plate was measured by a Laser Displacement Sensor (LK-G30, Keyence, Japan). The maximum acceleration of the plate is basically given by $\gamma=4 \pi^{2} f^{2} A$. Before shaking, the droplet was first 

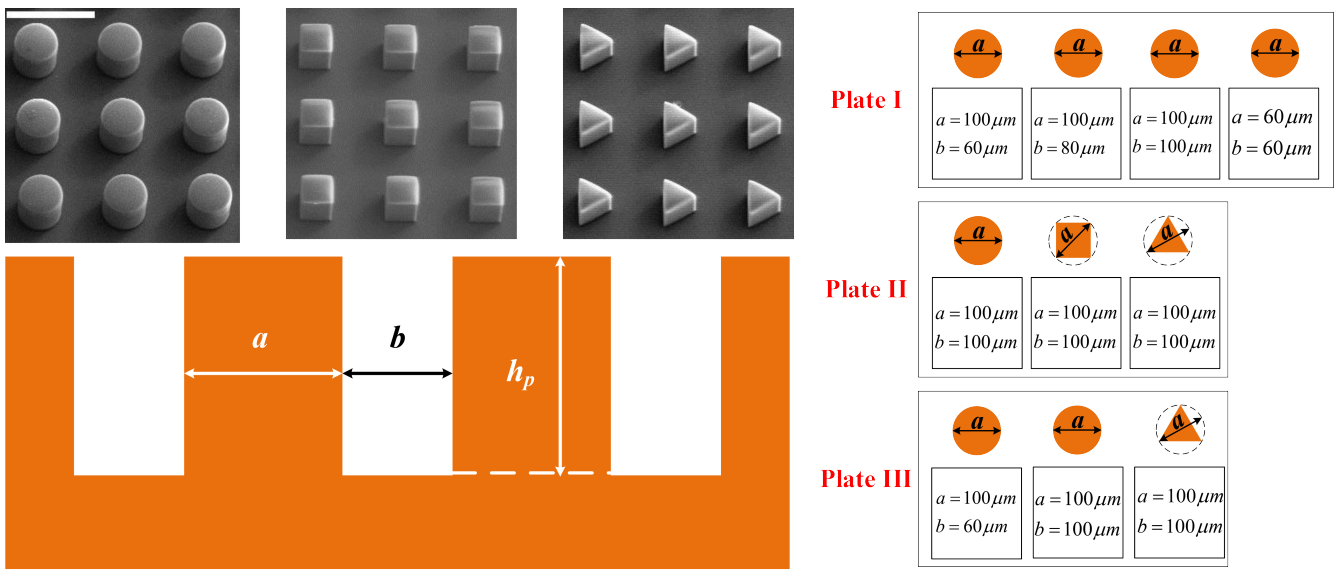

(a)

(b)

FiguRE 1. (a) Sketch of the micro-pillar patterns. The parameter $a, b$ and $h_{p}$ are the diameter of circumscribed circle to the pillar section, the distance between two neighboring pillars and the height of a pillar, respectively. The inserted pictures in (a) show the micro-pillars from the top (circular, squared and triangle). The scale bar is $200 \mu \mathrm{m}$. (b) Arrangement of the three plates.

studied on the substrate in a static mode in order to measure the contact diameter $d$ and the contact angle $\theta$. We tested three textured plates (Plate I, II and III) in the experiments, as shown in Fig. 1(b). These three plates were produced by Jiangsu Insitu Chip Technology Co., Ltd, China. Note that, Plate I and II were made of PDMS with the contact angle $\theta_{0}=105^{\circ}$, while Plate III was a textured silicon plate coated with hydrophobic membrane. According to the manufacturer, the contact angle of the hydrophobic membrane is $\theta_{0}=113^{\circ}$. The structural parameters of the lattice were tuned by varying the distance $b$ between the pillars and the size $a$ of the pillar. This latter parameter represents the diameter of the circumscribed circle to the pillar shape. The height $h_{p}$ of the pillars was fixed to $100 \mu \mathrm{m}$ for all the presented cases. Detailed parameters are presented in Table I. Concerning Plate I, four patterns with circular pillars were tested to investigate the influence of the area fraction $e$, i.e. the ratio between the top area of the pillars $s_{p}$ and that of the corresponding lattice cell $s_{c}=(a+b)^{2}$. On Plate II, three patterns with circular, squared and triangle pillars were implemented to show the influence of the pillar shape. The SEM pictures of the micro-pillars are shown in Fig. 1(a). The top area of one pillar was $\sqrt{3} a^{2} / 4, a^{2}$ and $\pi a^{2} / 4$ for the circular, squared and triangle pillar, respectively. Finally, on Plate III, 3 patterns with different area fractions and pillar shapes were tested to see the influence of the contact angle of the material $\theta_{0}$. Moreover, the theoretical apparent contact angle $\theta^{\star}$ was calculated using the formula $\cos \theta^{\star}=-1+e\left(1+\cos \theta_{0}\right)($ Cassie \& Baxter (1944)) and the actual contact angle $\theta$ was measured by analyzing the captured static images. In total, we conducted experiments on ten different patterned substrates.

\section{Results}

A typical experiment consisted in releasing a droplet on the substrate that was fixed to the shaker. Starting at rest, the frequency was fixed, the amplitude $A$ of the shaker was slowly increased. For low amplitudes, (Type I) the contact line remains pinned on the surface, while the water drop deforms at the frequency of the shaker. The contact angle cyclically changes during the deformation of the droplet. For larger amplitudes, (Type II) 


$\begin{array}{lcccccc}\text { Plate } & a(\mu \mathrm{m}) & b(\mu \mathrm{m}) & e & \theta_{0}\left({ }^{\circ}\right) & \theta^{\star}\left({ }^{\circ}\right) & \theta\left(^{\circ}\right) \\ \text { I } & 100 & 60 & 0.307 & 105 & 140.58 & 130 \pm 2 \\ \text { I } & 100 & 80 & 0.242 & 105 & 145.15 & 133 \pm 3 \\ \text { I } & 100 & 100 & 0.196 & 105 & 148.73 & 145 \pm 2 \\ \text { I } & 60 & 60 & 0.196 & 105 & 148.73 & 140 \pm 3 \\ \text { II } & 100 & 100 & 0.196 & 105 & 148.73 & 142 \pm 3 \\ \text { II } & 100 & 100 & 0.125 & 105 & 155.14 & 144 \pm 2 \\ \text { II } & 100 & 100 & 0.081 & 105 & 160.05 & 152 \pm 3 \\ \text { III } & 100 & 60 & 0.307 & 113 & 144.39 & 135 \pm 3 \\ \text { III } & 100 & 100 & 0.196 & 113 & 151.72 & 148 \pm 2 \\ \text { III } & 100 & 100 & 0.081 & 113 & 161.92 & 154 \pm 4\end{array}$

TABLE 1. Detailed parameters of the textured substrates

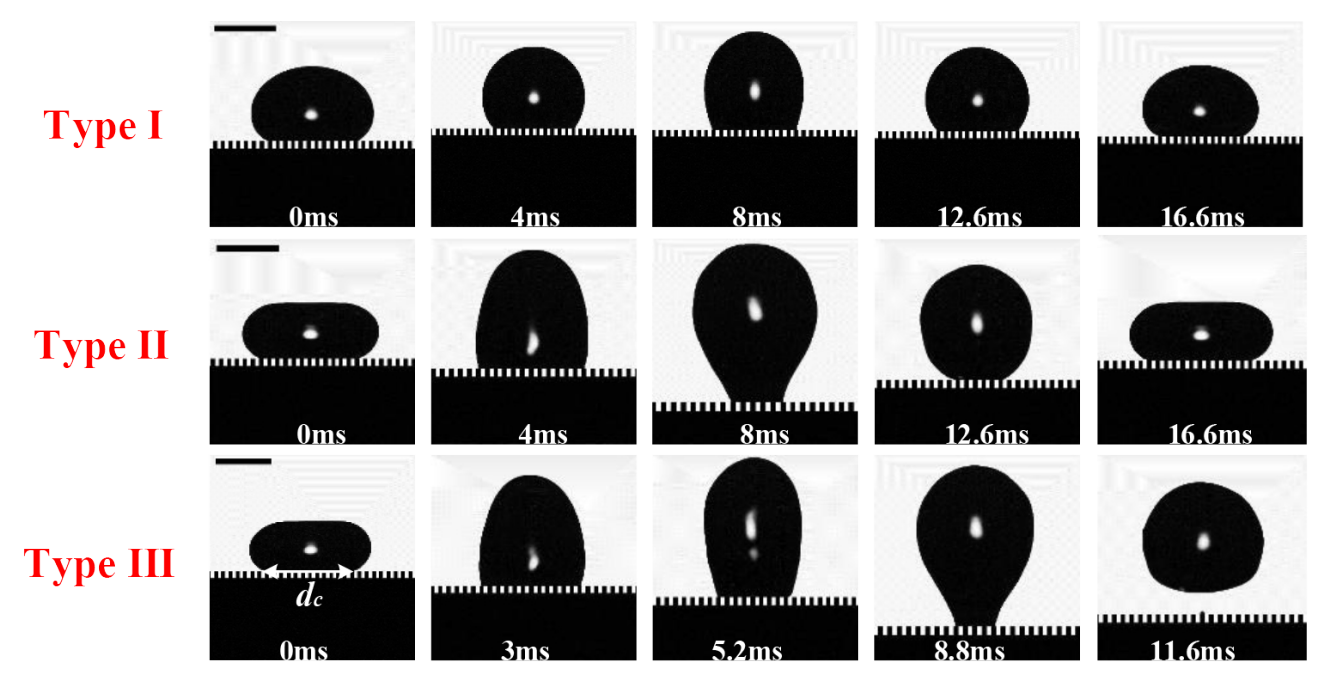

Figure 2. Different behaviors of the water drop $(D=2.02 \mathrm{~mm})$ on the vibrating textured surface at $60 \mathrm{~Hz}$. (I) The contact line remains pinned while the water drop deforms with oscillating contact angle; $d$ is constant. (II) The contact line oscillates with a constant amplitude; $d$ oscillates. (III) The water drop bounces off the textured surface; $d$ goes to zero. The scale bar indicates $1 \mathrm{~mm} . d_{c}$ is the maximal contact diameter before the separation. Note that, as we focus on the deformation of the water drop, position of the textured surface does not represent the actual height.

the contact area increases and decreases at the same frequency as the forced oscillation. The definitions of Type I and Type II follow these of Ref.(Noblin et al. (2004)). Let us note that, the droplet remains in the first mode of deformation. Finally, (Type III) the water drop bounces off the textured surface as $A$ reaches a critical value $A_{c}$. The three regimes are illustrated in Fig. 2 for a droplet of $2.02 \mathrm{~mm}$ of diameter and at a frequency $f=60 \mathrm{~Hz}$. In the following, we focus on the transition between Type II and Type III. More precisely, for each experimental set of parameters (droplet size, substrate, frequency), one measured the minimum acceleration $\gamma_{c}$ that allows the droplet to bounce off the plate. Indeed, let us note that when the droplet is bouncing, if the amplitude is decreased, the droplet still bounces even below the acceleration threshold $\gamma_{c}$ because the restitution coefficient is large exactly like in the bouncing ball problem.

To acknowledge the dynamics of water drop on the vibrating textured surface, we 

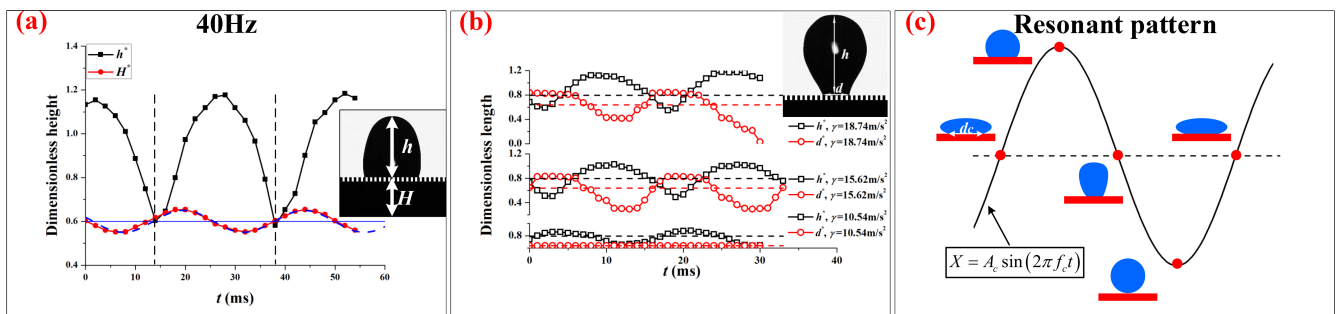

FiguRE 3. Dynamics of the water drop on the vibrating textured surface. (a) Time evolution of the water drop shape at the forced vibration frequency $f=40 \mathrm{~Hz}$.Here $h^{\star}$ and $H^{\star}$ are the dimensionless height normalized by the diameter of the droplet $D$. The blue dashed line is the fitted function graph in the form of a trigonometric function. The black dashed line is used for guide. (b) Time evolution of the droplet apparent diameter of contact $d$ with the plate and vertical extension of the droplet $h$ normalized by the diameter of the droplet $D=2.02 \mathrm{~mm}$. The frequency of excitation was $60 \mathrm{~Hz}$. Three cases are presented from bottom to top $\gamma=10.54,15.62$ and $18.74 \mathrm{~m} / \mathrm{s}^{2}$ that correspond to Type I, Type II and Type III, respectively. (c) Schematic drawing of water drop dynamics under the resonant vibration frequency, where $d_{c}$ is the maximal contact diameter before the separation and $X$ is the position of the plate with $X=0$ indicating the initial position.

measured the time evolution of the water drop height $h$. The dynamics of the textured surface was obtained by measuring the position $H$ of the plate. The trajectory of the plate $H^{\star}=H / D$ and the trajectory of the drop height $h^{\star}=h / D$ bouncing on a surface vertically, excited at $40 \mathrm{~Hz}$ are presented in Fig. 3(a). The drop diameter was $D=2.62 \mathrm{~mm}$ and the textured surface was made of triangle top face pillars characterized by $e=0.081$. The trajectory of the textured surface was fitted by a trigonometric function (the blue dashed lines in the picture). One can see that the droplet impacted the plate once per period; this is the first bouncing mode. Figure 3(b) presents the evolution of the normalized droplet apparent diameter of contact $d^{\star}=d / D$ with the plate and the normalized droplet height $h^{\star}=h / D$. The frequency was then fixed at $60 \mathrm{~Hz}$. Three situations are presented on the Figure, i.e. for three accelerations of the plate $\gamma=4 \pi^{2} A f^{2}=10.54$ (Type I), 15.62 (Type II) and $18.74 \mathrm{~m} / \mathrm{s}^{2}$ (Type III) for the bottom, middle and top curves, respectively. One can see that, for the Type I, $d^{\star}$ remains constant while the droplet deforms in phase with the plate. For the Type II, The contact line oscillates and consequently $d^{\star}$. One notes that $d^{\star}$ and $h^{\star}$ oscillate at opposite phase, this is the first eigenmode of the droplet. Finally, for its highest shown acceleration, $d^{\star}$ went to zero. This is the signature of the detachment (Type III). To summarize, one proposes a schema that stands for the phenomenology of the bouncing (Fig. 3(c)). When the water droplet height reaches the minimum (or the maximal deformation), the water droplet velocity is equivalent to the textured surface. At the resonance, the water droplet reaches the maximal deformation when the textured surface moves upwards with the highest speed. In this way, the water droplet can pick up the maximal kinetic energy during one vibration period. Subsequently, the water droplet retracts and undergoes a vertical elongation. At last, the water droplet evolves into a sphere ball and rightly separates with the textured surface at the lowest position. At this point, the textured surface has the highest upward acceleration which drives the water droplet to vertically deform till the maximal deformation.

The dependence of the acceleration threshold for the take-off was studied on the triangle pillar with $e=0.081$, i.e. for the lowest tested value of the parameter $e$. Under this condition, the contact area between the droplet and the substrate is minimized. The values of the thresholds are reported in Fig. 4(a) for different sizes of droplets (between $D=2.02$ and $2.62 \mathrm{~mm}$ ) as a function of the excitation frequency. The horizontal dashed 
line represents the Earth gravity acceleration. The critical acceleration for the take-off of the droplet as a function of the frequency follows parabolic shape curves that are characteristic of resonant behavior (Hubert et al. (2015)). The minimum occurs at a frequency $f_{c}$ and an acceleration value $\gamma_{c}=4 \pi f_{c}^{2} A_{c}$ where $A_{c}$ is the critical amplitude of the oscillation for which the droplet takes off. In the inset of Fig. 4(a), one reports the resonant frequency $f_{c}$ as a function of the droplet diameter for different surfaces made of PDMS in a log-log plot. The solid line corresponds to a slope of $3 / 2$, that means that one finds that $f_{c} \sim D^{-3 / 2}$. This is in good agreement with the scaling of the eigenfrequency of the droplet mode (Rayleigh (1879)). Moreover, the resonant frequency was found to increase with the area fraction. In Fig. 4(b), the normalized threshold amplitude $A_{c}^{\star}=A_{c} / D$ is reported as a function of the frequency $f$ normalized by the experimental critical frequency $f_{c}$. One finds that all the data collapse on a same curve, meaning that $A_{c} \propto D$. The dashed curve (Fig. 4(b)) corresponds to a fit using Eq. (8) in Ref. (Hubert et al. (2015)). All in all, the critical acceleration $\gamma_{c}$ depends on the diameter of the droplet as $D^{-2}$, i.e. the larger the droplet, the smaller the acceleration required for the take-off, as shown in Fig. 4(a). Surprisingly, it is easier to make a big droplet to take off than a small one. A priori, two mechanisms should lead to the opposite conclusion: a viscous mechanism (the larger droplets dissipate more than the smaller) (de Ruiter et al. (2015)) and the adhesion force (a large droplet touches the plate with a larger area than the smaller one) (Olin et al. (2013); Butt et al. (2017)). On the other hand, the large droplet deforms much more easily than the small ones and allows the take-off even for accelerations below $g$. Figure 4(c) shows the critical acceleration as a function of the frequency for five different surfaces that differ from the pattern structure of the pillars and from the distance between the pillars for the circular patterns. The size $D$ of the drop was $2.62 \mathrm{~mm}$. The different substrates are here summarised by the only parameter $e$. The shapes of the pillars (circular, squared or triangle) are represented by symbols that have the same shape. The critical acceleration for the take-off of the droplet again follows parabolic shape curves characteristic of the resonance. One measured that both $\gamma_{c}$ and $f_{c}$ increase with the solid fraction $e$. In Ref. (Sharp et al. (2011); Sharp (2012)), the resonant frequency of a sessile droplet whose mass is fixed is found to increase when the contact angle decreases in the hydrophobic case (i.e. $\theta>90^{\circ}$ ). In our case, we measured that when the solid fraction $e$ is increased from 0.081 to 0.307 the contact angle decreases from $150^{\circ}$ to $130^{\circ}$. According to Ref.(Sharp et al. (2011)), using Eq.(4), one finds that the resonant frequency of a sessile droplet is proportional to $\sqrt{\left(\cos ^{3} \theta-3 \cos \theta+2\right) / \theta^{3}}$. Consequently, the ratio of the resonant frequencies corresponding to contact angles of $150^{\circ}$ to $130^{\circ}$ is found to be 1.19 which is not far from the ratio found from our measurements $46 / 40=1.15$ . In total, we can understand the resonant frequency shift towards higher values when $e$ increases, see the inset of Fig. 4(a).

As for $\gamma_{c}$, its value decreases with the solid fraction $e$. That strongly suggests that the adhesion is proportional to the contact area. On the one hand, one finds that $\gamma_{c}$ is lower than the gravity acceleration for $e=0.081$. Such low accelerations for the take-off are only possible because of the deformation of the droplet (Hubert et al. (2015)). On the other hand, one can infer that the threshold is related to the energy required to break all the capillary bridges between the droplet and the textured surface. In Fig. 5, snapshots present the separation between the water and the textured surface. For the sake of observation, we captured the dynamics of a water drop bouncing off the static textured surface. The corresponding drawings indicate the time evolution of the capillary bridge which are believed to dominate the energy dissipation (Olin et al. (2013); Butt et al. (2017)). As the water drop retracts, the contact angle first decreases. A capillary bridge still links the droplet to the pillar before the contact line slips off the top face of the 
(a)

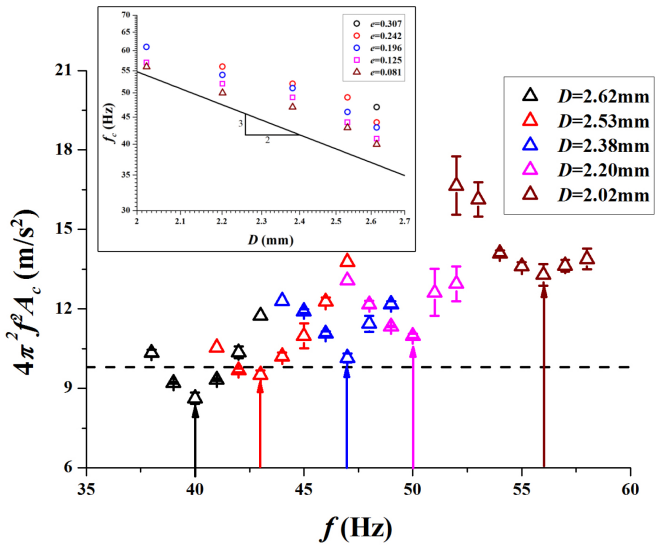

(b)

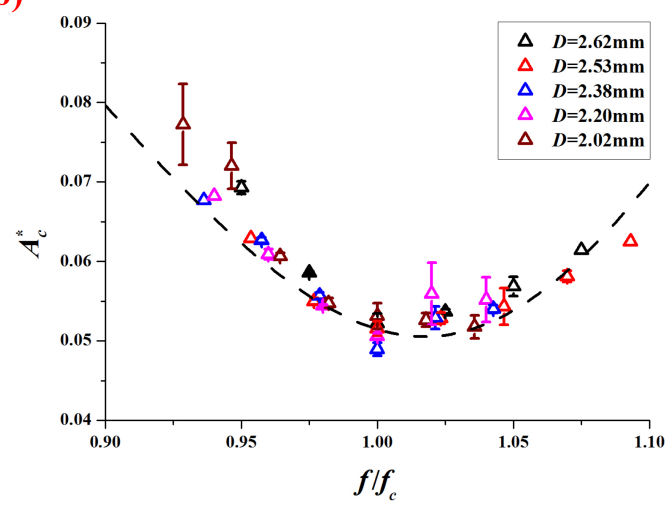

(c)

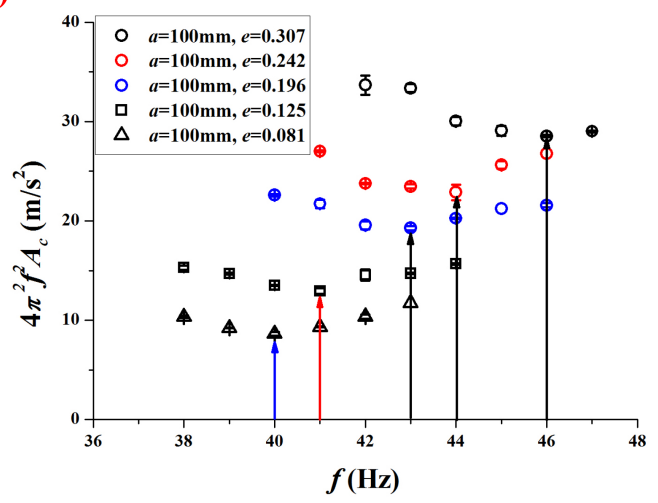

Figure 4. (a) The critical acceleration required for the water drop bouncing off the textured surface as a function of the frequency for 5 different sizes of droplet. The arrows indicate the minima and the corresponding frequencies are the resonant frequencies. These data were obtained using the texture surface with triangle top face $(a=100 \mu \mathrm{m}, e=0.081)$. The black dashed line indicates the value of the gravity acceleration $g$. Inset: Dependance of the resonant frequency against the drop diameter. The solid line indicates $f_{c} \sim D^{-3 / 2}$. (b) Normalized threshold amplitude vary with the frequency $f$ divided by the experimental resonant frequency $f_{c}$. The dashed curve corresponds to a fit coming from Hubert et al. (2015). (c) Critical acceleration for take-off as a function of the frequency for 5 different surfaces characterized by the parameter $e$. Circular, square and triangle pillars surfaces were investigated and are represented by symbols of the corresponding shape. The frequency $f_{c}$ and the acceleration $4 \pi f^{2} A_{c}$ at which the minimum occurs are found to increase with the solid fraction $e$. 


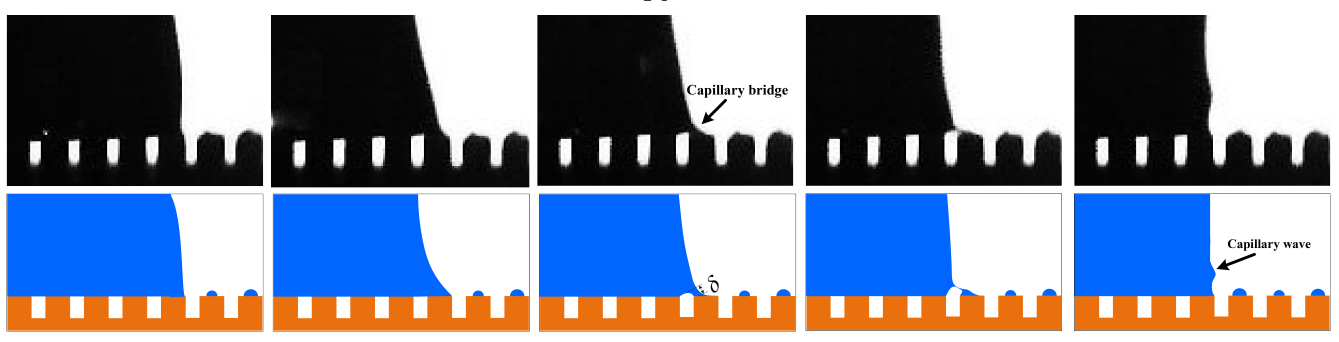

FIGURE 5. Experimental images and schematics of the evolution of a capillary bridge. Pinch-off of the capillary bridge leaves a tiny water drop on the top face of the micro-pillar. Meanwhile, a capillary wave is generated and penetrates upwards, undergoing viscous dissipation.

micro-pillar. Then the bridge pinches off, leaving a tiny water drop on the micro-pillar and giving rise to a capillary wave which undergoes viscous dissipation. In total, the energy stored in the capillary bridge is dissipated, instead of being converted into kinetic energy (Olin et al. (2013); Butt et al. (2017)). Considering the high restitution coefficient of the bouncing water drop, viscous dissipation caused by the drop deformation should be negligible (Richard \& Quéré (2000); de Ruiter et al. (2014, 2015)). Moreover, the restitution coefficient of bouncing water drop is significantly lower than 1 because of the drop oscillation after lift-off (Richard \& Quéré (2000)). Under the presented conditions, especially at resonance, the water drop detaches from the substrates almost in the shape of a sphere, see Fig. 2 (Type III) and the drawing in Fig. 3(c).

The capillary bridge is modelled by a linear spring. The adhesion energy $E_{a d h}$ depends on the maximum extension $\delta_{m}$ of the bridge before breaking and the maximum number $N$ of pillars that the droplet covers during one oscillation. The elastic energy $E_{e}$ stored in one bridge is given by $E_{e} \sim \sigma \delta^{2}$, where $\sigma$ and $\delta$ are the surface tension of water and the extension of the capillary bridge, respectively. The maximal extension before the pinch-off is proportional to the diameter of the micro-pillar, i.e. $\delta_{m} \sim a \cos ^{2}\left(\theta_{0} / 2\right)$ (Butt et al. (2017)). The total number $N$ of capillary bridges to overcome is given by $\pi d_{c}^{2} e / 4 s_{p}$ , where $d_{c}$ is the maximal diameter of the apparent contact area between the droplet and the substrate (as shown in Fig. 2 (Type III)). Thus the adhesion energy $E_{a d h}=N E_{e}$ for the detachment scales with $\sigma d_{c}^{2} e \cos ^{4}\left(\theta_{0} / 2\right)$. To determine $d_{c}$, one considers that the maximum of deformation occurs when the textured surface accelerates upwards, leading to the vertical deformation of the water droplet. A similar phenomenon has been reported in the previous literature (Raufaste et al. (2017); Clanet et al. (2004)). Considering that the droplet has a cylinder shape with a height $h$, one finds that $h d_{c}^{2} \sim D^{3}$. When submitted to gravity solely, the droplet height $h$ is given by $2 \ell_{c} \sin \theta / 2$ where $\ell_{c}=\sqrt{\sigma / \rho g}$ (de Gennes et al. (2008)). Here, one takes into account the maximum acceleration of the droplet during the oscillations, i.e. $4 \pi^{2} f_{c}^{2} A_{c}$ and one defines the effective capillary length is $\ell_{c}=\sqrt{\sigma / \rho\left(g+4 \pi^{2} f_{c}^{2} A_{c}\right)}$. In the range of the considered parameters, one can neglect (see later) the role of $g$ keeping solely the dependence of the adhesion with the acceleration of the plate and with the diameter of the droplet, i.e. $h=2 \sqrt{\sigma / \rho\left(4 \pi^{2} f_{c}^{2} A_{c}\right)} \sin \theta / 2$. Although deviations can be observed between $\theta^{\star}$ and $\theta$, we still prefer to express the contact angle as $\cos \theta=-1+e\left(1+\cos \theta_{0}\right)$ (Cassie \& Baxter (1944)). This latter relation can be injected into the expression of $h$ after some trigonometric manipulations. One obtains

$$
h=2 \sqrt{\frac{\sigma}{4 \pi^{2} f_{c}^{2} \rho A_{c}}} \sqrt{1-\frac{e}{2}\left(1+\cos \theta_{0}\right)}
$$


namely

$$
d_{c}^{2} \sim D^{3} \sqrt{\frac{\rho f_{c}^{2} A_{c} / \sigma}{1-e\left(1+\cos \theta_{0}\right) / 2}}
$$

Finally, we obtain the energy dissipation during the separation $E_{a d h} \sim D^{3} e\left(\rho \sigma f_{c}^{2} A_{c}\right)^{1 / 2}[1-$ $\left.e\left(1+\cos \theta_{0}\right) / 2\right]^{-1 / 2} \cos ^{4}\left(\theta_{0} / 2\right)$. The energy that propels the droplet finds its origin in both the kinetic energy provided by the plate $E_{k} \sim \rho D^{3} f_{c}^{2} A_{c}^{2}$ and by the energy stored in the surface of the droplet $\Delta E_{s}$. Analogous to the impacting drop on a solid surface, the stored surface energy should be $\Delta E_{s} \sim \rho D^{3} f_{c}^{2} A_{c}^{2}$. Thus the total energy of the water drop is $E_{w}=E_{k}+\Delta E_{s} \sim \rho D^{3} f_{c}^{2} A_{c}^{2}$. Balancing $E_{w}=E_{a d h}$, we obtain the critical condition for the water drop bouncing off the textured surface $f_{c} A_{c}^{3 / 2} \sim C_{\theta}(\sigma / \rho)^{1 / 2}$ with the constant $C_{\theta}=e\left[1-e\left(1+\cos \theta_{0}\right) / 2\right]^{-1 / 2} \cos ^{4}\left(\theta_{0} / 2\right)$. Consequently, since we have that the critical frequency scales with the eigenfrequency of the fundamental droplet mode $\sqrt{\sigma / \rho D^{3}}$, one finds

$$
A_{c} \sim C_{\theta}^{2 / 3} D
$$

other parameters being non-dimentional pre-factors. The later expression is independent of the surface tension. Let us note that, if we keep the dependence of $g$ in the effective capillary length, one finds that the relationship between $A_{c}$ and $D$ starts to slightly deviate from the linear behaviour for large diameters. As the range of investigated droplet diameters is reduced, it is not possible to evidence this slight non linear behaviour. In addition, the eigenfrequency of the droplet increases when the volume of the droplet increases. As a consequence, the scaling presented in Eq. (3.1) works very well for small droplets as the critical acceleration increases making the influence of the gravity less and less important. In Fig. 6(a), the values of $A_{c}$ are plotted as a function of the diameter of the droplet $D$ and that for the different tested configuration. The data sets concerning the same value of $e$ aligns and can be fitted by the proposed scaling $A_{c}=\beta D$. The proportional factor $\beta$ is found to be about $5 \%$ for the substrate made of PDMS with $e=0.081$. That means that, at resonance, the droplet detaches when the amplitude of the shaker surpasses $5 \%$ of the droplet diameter. The value of $\beta$ are reported as a function of $C_{\theta}^{2 / 3}$ in Fig. 6(b). The data points again align confirming the prediction Eq.(3.1). The linear dependence of $A_{c}$ with the diameter of the droplet $D$ is only logical since one needs to inject more energy $\left(\propto A^{2} f^{2}\right)$ to make the droplet to detach when the droplet is large. On the other hand, the critical frequency is related to the eigenmode frequency of the droplet and scales like $D^{-3 / 2}$. The acceleration of the plate depends on $f^{2}$ and then the critical acceleration on $D^{-3}$. In consequence, a larger acceleration of the plate is required to excite the eigenmode of the droplet and to reach the critical amplitude. Small droplets are more difficult to take off egarding the maximum acceleration.

\section{Conclusion}

small droplets

The dynamics of a water drop detaching from the vibrating textured surfaces has been studied. Experiments were conducted on textured surfaces made of pillar patterns of different shapes and of different geometrical arrangements. The results allow to determine the acceleration threshold required to detach a droplet that was initialized in the CassieBaxter state. The acceleration threshold of the plate to allow the take-off depends on the frequency, the pattern configuration of the substrate and the droplet diameter. The minimal acceleration for the take-off was found to be larger for small droplets than for large droplets. Moreover, this minimal acceleration occurs at a critical frequency that scales with the eigenmode of the vibration of the droplet. On the other hand, the 
(a)

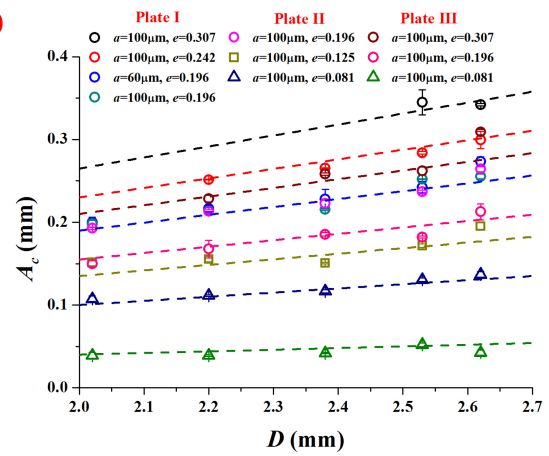

(b)

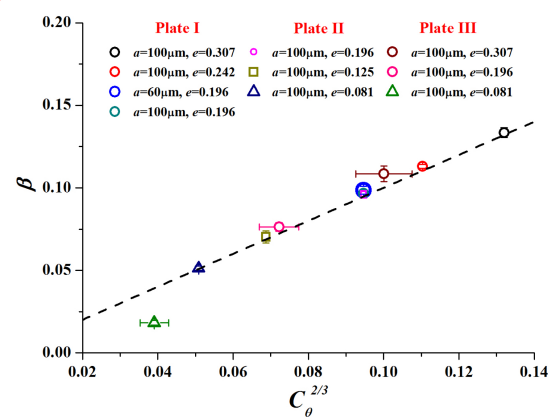

FiguRE 6. (a) Threshold for detaching a water drop from the textured surface. Experimental results are fitted with the corresponding dashed lines $A_{c}=\beta D$. (b) Relationship between $\beta$ and $C_{\theta}^{2 / 3}$. To distinguish the three coincident cases with $e=0.196$, the corresponding symbols are presented in different sizes.

threshold for the amplitude of vibration was found to be proportional to the diameter of the droplet. The developed model is based on the energy required to break the capillary bridges between the micro-pillars and the droplet. Giving the solid fraction $e$ and the contact angle $\theta_{0}$ on the smooth surface, this simple model allows to explain why small droplets required a larger acceleration to take-off than larger one.

\section{Acknowlegements}

We acknowledge support from the National Natural Science Foundation (Grant No. 51875507). S. Dorbolo is a Senior Research Associate F.R.S.-FNRS. Special thanks to Prof. Jinyou Shao and Dr. Chunhui Wang of Xi'an Jiaotong University, for the design and manufacture of the textured surfaces. The authors also thank I. Ashraf and C. Marique for the careful reading of the text.

\section{REFERENCES}

Bartolo, D., Josserand, C. \& Bonn, D. 2005 Retraction dynamics of aqueous drops upon impact on non-wetting surfaces. Journal of Fluid Mechanics $\mathbf{5 4 5}$ (-1), 329.

Boreyko, J. B. \& Chen, C. H. 2009 Restoring superhydrophobicity of lotus leaves with vibration-induced dewetting. Physical Review Letter 103 (17), 174502.

Brunet, P., Eggers, J. \& Deegan, R. D. 2007 Vibration-induced climbing of drops. Physical Review Letter 99 (14), 144501.

Butt, H. J., Gao, N., Papadopoulos, P., Steffen, W., Kappl, M. \& Berger, R. 2017 Energy dissipation of moving drops on superhydrophobic and superoleophobic surfaces. Langmuir 33 (1), 107-116.

Cassie, A. B. D. \& Baxter, S. 1944 Wettability of porous surfaces. Transactions of the Faraday Society 40 (0), 546-551.

Clanet, C., BÉGuin, C., Richard, D. \& QuÉRÉ, D. 2004 Maximal deformation of an impacting drop. Journal of Fluid Mechanics 517, 199-208.

Couder, Y., Fort, E., Gautier, C. H. \& Boudaoud, A. 2005 From bouncing to floating: noncoalescence of drops on a fluid bath. Physical Review Letter 94 (17), 177801.

Daniel, S., Chaudhury, M. K. \& De Gennes, P. G. 2005 Vibration-actuated drop motion on surfaces for batch microfluidic processes. Langmuir 21 (9), 4240-4248.

De Gennes, P. G., Brochard-Wyart, F. \& Quéré, D. 2008 Capillary and wetting phenomena. Springer, New York . 
Gilet, T., Terwagne, D., Vandewalle, N. \& Dorbolo, S. 2008 Dynamics of a bouncing droplet onto a vertically vibrated interface. Physical Review Letter 100 (16), 167802.

Hubert, M., Robert, D., Caps, H., Dorbolo, S. \& Vandewalle, N. 2015 Resonant and antiresonant bouncing droplets. Physical Review E 91 (2), 023017.

Khojasteh, D., Kazerooni, M., Salarian, S. \& Kamali, R. 2016 Droplet impact on superhydrophobic surfaces: A review of recent developments. Journal of Industrial and Engineering Chemistry 42, 1-14.

Kim, H. \& Hee-Chang, L. 2015 Mode pattern of internal flow in a water droplet on a vibrating hydrophobic surface. The Journal of Physical Chemistry B 119 (22), 6740-6746.

MaO, T., Kunn, D. CS \& Tran, H. 1997 Spread and rebound of liquid droplets upon impact on flat surfaces. AIChE Journal 43 (9), 2169-2179.

McBride, S. A., Dash, S. \& Varanasi, K. K. 2018 Evaporative crystallization in drops on superhydrophobic and liquid-impregnated surfaces. Langmuir 34 (41), 12350-12358.

Noblin, X., Buguin, A. \& Brochard-Wyart, F. 2004 Vibrated sessile drops: transition between pinned and mobile contact line oscillations. The European Physical Journal E 14 (4), 395-404.

Olin, P., Lindstrom, S. B., Pettersson, T. \& Wagberg, L. 2013 Water drop friction on superhydrophobic surfaces. Langmuir 29 (29), 9079-89.

QuÉRÉ, D. \& REYssat, M. 2008 Non-adhesive lotus and other hydrophobic materials. Philosophical Transactions of the Royal Society A 366 (1870), 1539-1556.

Raufaste, C., Chagas, G. R., Darmanin, T., Claudet, C., Guittard, F. \& Celestini, F. 2017 Superpropulsion of droplets and soft elastic solids. Physical Review Letter 119 (10), 108001.

Rayleigh, Lord 1879 Vi. on the capillary phenomena of jets. Proceedings of the Royal Society of London 29 (196-199), 71-97.

Richard, D., Clanet, C. \& Quéré, D. 2002 Surface phenomena: Contact time of a bouncing drop. Nature 417 (6891), 811.

RICHARD, D. \& QuÉRÉ, D. 2000 Bouncing water drops. EPL (Europhysics Letters) 50 (6), 769.

De Ruiter, J., Lagraauw, R., van den Ende, D. \& Mugele, F. 2014 Wettabilityindependent bouncing on flat surfaces mediated by thin air films. Nature Physics 11 (1), $48-53$.

De Ruiter, J., Lagraauw, R., Mugele, F. \& Van den Ende, D. 2015 Bouncing on thin air: how squeeze forces in the air film during non-wetting droplet bouncing lead to momentum transfer and dissipation. Journal of Fluid Mechanics 776, 531-567.

Sharp, J. S. 2012 Resonant properties of sessile droplets; contact angle dependence of the resonant frequency and width in glycerol/water mixtures. Soft Matter 8 (2), 399-407.

Sharp, J. S., Farmer, D. J. \& Kelly, J. 2011 Contact angle dependence of the resonant frequency of sessile water droplets. Langmuir 27 (15), 9367-71.

Smith, J. D., Dhiman, R., Anand, S., Reza-Garduno, E., Cohen, R. E., McKinley, G. H. \& VARANASI, K. K. 2013 Droplet mobility on lubricant-impregnated surfaces. Soft Matter 9 (6), 1772-1780.

Wei, L., Zhinai, J., H., Jichang, C., Taimin \& Gang, W. 2014 Vibration-induced wenzelcassie wetting transition on microstructured hydrophobic surfaces. Applied Physics Letters 104 (18), 181601.

Zawala, J., Dorbolo, S., Terwagne, D., Vandewalle, N. \& Malysa, K. 2011 Bouncing bubble on a liquid/gas interface resting or vibrating. Soft Matter 7 (14), 6719-6726. 